\title{
GUIDELINES DE CONTROLADORES PID PARA CICLO DE REFRIGERAÇÃO INDUSTRIAL
}

\author{
S. B. B. L. VILLAR ${ }^{1}$, T. G. NEVES ${ }^{1}$, J. I. H. T. NETO ${ }^{2}$ e A. C. B. ARAÚJO ${ }^{1}$ \\ ${ }^{1}$ Universidade Federal de Campina Grande, Unidade Acadêmica de Engenharia Química \\ ${ }^{2}$ Braskem S/A \\ E-mail para contato: savana_lelis@hotmail.com
}

\begin{abstract}
RESUMO - O controlador PID é o algoritmo de controle mais difundido nas indústrias em todo o mundo, além de estar disponível em quase todas as plataformas de controle comerciais. No entanto, algumas orientações gerais precisam ser consideradas na tomada de decisões relativas à aplicação do PID para uma determinada exigência de controle. Portanto, o objetivo deste trabalho foi simular um sistema de refrigeração industrial e algumas estratégias de controle. Analisaram-se as três ações básicas do controlador PID, realizando-se uma comparação entre as ações isoladas e combinadas. Por fim, os resultados obtidos mostram que com o uso do controlador PID a variável é corrigida da melhor maneira possível, quando comparado aos controladores P e PI, onde a estabilização no novo regime acontece de forma rápida, apresentando um offset praticamente nulo e um desvio pouco acima do valor desejado.
\end{abstract}

\section{INTRODUÇÃO}

O presente trabalho estuda a simulação de um sistema de refrigeração industrial e a importância das estratégias de controle. Estudar as diferentes configurações de controladores PID (Proporcional, Integral e Derivativo) em um sistema de refrigeração significa atender aos anseios de diversas indústrias, onde a refrigeração é utilizada para viabilizar processos variados.

É notório que o controle de processos represente uma área de estudo muito importante nas indústrias, pois o interesse de manter o processo em seus pontos operacionais mais eficientes traz benefícios não somente econômicos, mas também previne risco para as pessoas e equipamentos.

\section{IMPORTÂNCIA DO CONTROLADOR PID}

Termos como controle de qualidade, controle de finanças, controle de produção, entre outros, cobrem um vasto espectro de atividades. Todos estes contextos estão baseados na existência de um sistema, cujo comportamento se queira influenciar, e na liberdade de tomar ações, as quais irão forçar o sistema a agir de maneira desejada (Bissel, 1993).

Uma das estruturas mais utilizadas na implementação de controladores industriais é a PID, por tratar-se de uma das técnicas existentes de melhor custo/benefício: fácil implementação, 
baixo custo, número reduzido de parâmetros a serem sintonizados, além de um desempenho bastante satisfatório em vários casos práticos (Qin e Badgwell, 2003). Os trabalhos de Aström e Hägglund (2001) mostraram que mais de $90 \%$ das malhas de controle industriais são do tipo PID.

\section{SISTEMA DE CONTROLE PID}

Normalmente, a função PID é usada para controlar as variáveis do processo, tais como temperatura, pressão, nível de líquido. O controlador PID recebe a variável de processo (PV) e controla a variável manipulada (MV) ajustando a PV para coincidir com o valor ajustado (SV).

A idéia básica por trás de um controlador PID é ler um sensor, calcular a resposta de saída do atuador através do cálculo das três ações e somar os três componentes para calcular a saída.

\section{GUIA RÁPIDO DE REFERÊNCIA DAS AÇÕES PROPORCIONAL, INTEGRAL E DERIVATIVA}

O efeito de cada uma das ações de controle no sistema em malha-fechada é sumariado na Tabela 1, admitindo como sinal de referência um degrau com amplitude qualquer.

Tabela 1 - Condições experimentais.

\begin{tabular}{ccccc}
\hline Ganhos & Tempo de subida $\left(\mathbf{t}_{\mathbf{r}}\right)$ & Sobre-sinal $\left(\mathbf{M}_{\mathbf{p}}\right)$ & $\begin{array}{c}\text { Tempo de } \\
\text { Estabilização }\left(\mathbf{t}_{\mathbf{s}}\right)\end{array}$ & $\begin{array}{c}\text { Erro de } \\
\text { Regime }\left(\mathbf{e}_{\mathbf{s s}}\right)\end{array}$ \\
\hline $\mathrm{K}_{\mathrm{p}}$ & Diminui & Aumenta & Pequena Alteração & Diminui \\
$\mathrm{K}_{\mathrm{i}}$ & Diminui & Aumenta & Aumenta & Elimina \\
$\mathrm{K}_{\mathrm{d}}$ & Pequena Alteração & Diminui & Diminui & Pequena Alteração \\
\hline
\end{tabular}

Esta tabela deverá ser empregada somente como um guia rápido de referência.

\subsection{Quando usar as ações PID isoladas e combinadas?}

As seguintes orientações gerais são sugeridas na tomada de decisões relativas à aplicação dos controladores PID para uma determinada exigência de controle:

- Controlar a temperatura da água gelada ou construção de pressões estáticas são situações onde offsets grandes podem gerar problemas operacionais significativos, podendo ser eliminados via ação integral.

- Pensar bem antes de acrescentar ação derivativa em uma malha de controle. Para ser eficaz, deve ser aplicada com muito cuidado e bons ajustes. (Astrom e Hagglund, 2001).

- Observar o erro quando o setpoint é alterado. Se a ação integral prosseguir, haverá um atraso de tempo significativo em resposta ao overshoot (Corripio, 1990). 
- A ação de controle D raramente é utilizada em processos que têm um tempo de resposta rápido (Luyben, 1996).

- Em controle de nível, procurar usar apenas ação proporcional e, quando possível, com banda morta. (Skogestad, 2003).

\section{CICLO DE REFRIGERAÇÃO INDUSTRIAL}

A refrigeração é definida como o ramo da ciência que trata dos processos de redução e conservação da temperatura de um espaço ou material, abaixo da temperatura do ambiente circundante (Dossat, 2004).

Os ciclos de refrigeração são ciclos termodinâmicos que constituem o modelo matemático que define o funcionamento das máquinas frigoríficas e das bombas de calor.

\section{METODOLOGIA}

\subsection{O ciclo de Refrigeração Proposto}

A unidade fabril de Vinílicos localizada em Maceió - AL utiliza-se de unidades geradoras de água gelada que em baixa temperatura, é utilizada em diferentes processos. A Figura 1 mostra o ciclo de refrigeração simulando o ciclo industrial real no simulador Dynsim.

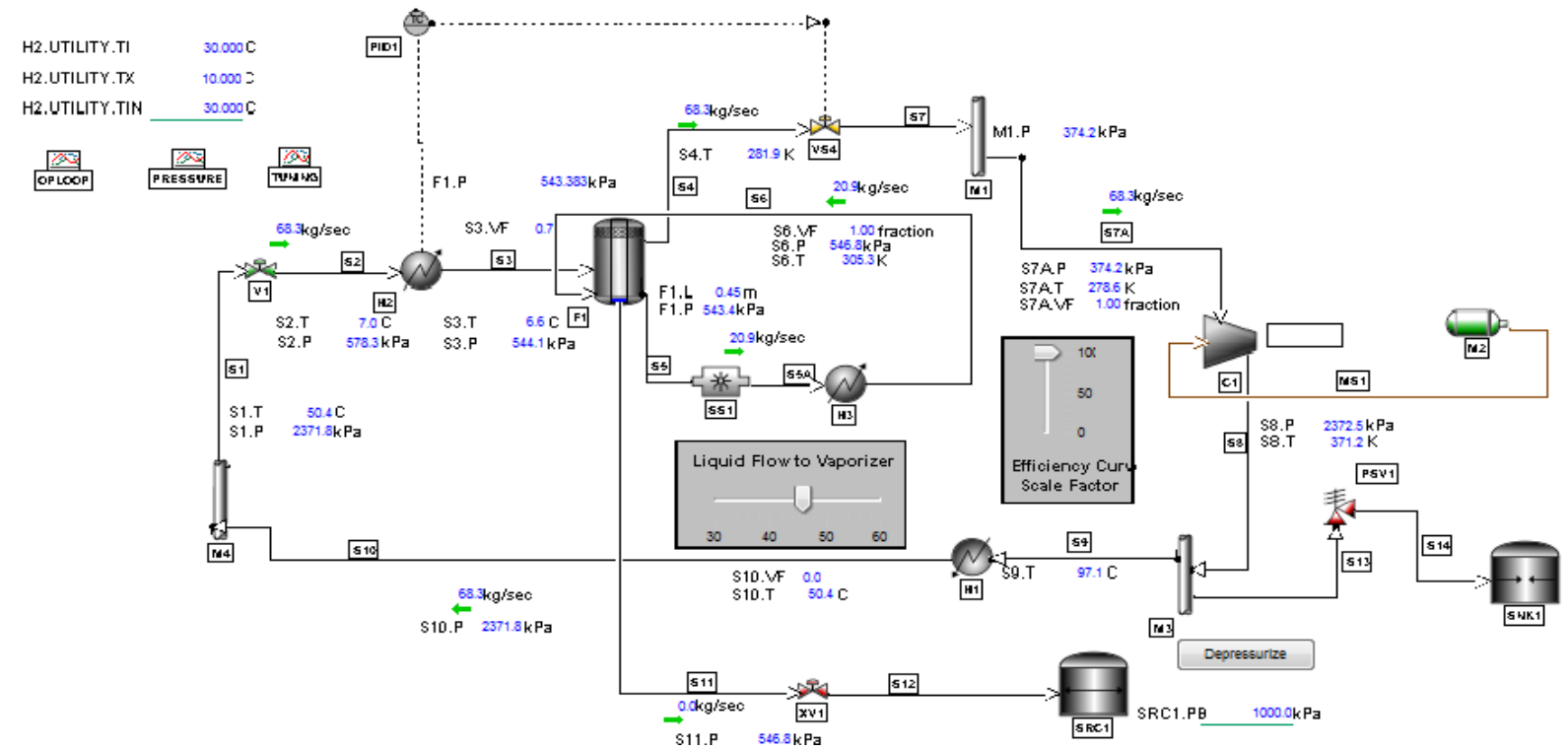

Figura 1 - Ciclo de refrigeração (DYNSIM V5. 1).

O vapor do refrigerante é comprimido e, em seguida, parcialmente condensado. A mistura passa pela válvula para ganhar calor através do trocador de calor novamente. Uma mistura de hidrocarbonetos leves que consiste principalmente em propano é utilizado como refrigerante. 


\section{RESULTADOS E DISCUSSÕES}

\subsection{Sintonia do PID}

Com o auxílio do programa Matlab tornou-se possível determinar a função de transferência do processo. Através do método de Ziegler-Nichols determinou-se os parâmetros do controlador, que são apresentados na Tabela 2 e as condições iniciais do processo na Tabela 3.

Tabela 2 - Valores de sintonia dos parâmetros do controlador PID

\begin{tabular}{cc}
\hline Parâmetro & Valor após sintonia \\
\hline $\mathrm{K}_{\mathrm{p}}$ & 1,067 \\
$\mathrm{~T}_{\mathrm{i}}$ & 60 \\
$\mathrm{~T}_{\mathrm{d}}$ & 15 \\
\hline
\end{tabular}

Tabela 3 - Condições iniciais do processo

\begin{tabular}{cc}
\hline Variável & Valor \\
\hline $\begin{array}{c}\text { Temperatura da água na entrada } \\
\text { do evaporador } \\
\text { Set-Point }\end{array}$ & $30^{\circ} \mathrm{C}$ \\
& $10^{\circ} \mathrm{C}$ \\
\hline
\end{tabular}

\subsection{Análise das Respostas Dinâmicas Frente a Distúrbios}

Foram perturbadas duas variáveis na planta. As propostas estão apresentadas na Tabela 4.

Tabela 4 - Proposta de distúrbios na planta

\begin{tabular}{ccc}
\hline Estratégia & Variável Controlada & Variável Manipulada \\
\hline I & $\begin{array}{c}\text { Temperatura da água à saída do } \\
\text { evaporador }(\mathrm{H} 2)\end{array}$ & $\begin{array}{c}\text { Fluxo mássico de fluido } \\
\text { refrigerante na corrente } \mathrm{S} 7\end{array}$ \\
II & $\begin{array}{c}\text { Temperatura da água à saída do } \\
\text { evaporador }(\mathrm{H} 2)\end{array}$ & $\begin{array}{c}\text { Fluxo mássico de fluido } \\
\text { refrigerante na corrente } \mathrm{S} 11\end{array}$ \\
\hline
\end{tabular}

É importante compreender as respostas dinâmicas do modelo por variação das condições em que a planta é operada. Para isso, os seguintes casos foram analisados.

A fim de analisar a influência da diminuição da vazão mássica de refrigerante no ciclo, alterou-se a abertura da válvula VS4. Pode-se observar que quando a vazão mássica é diminuída, a capacidade de refrigeração do evaporador cai como mostrado na Figura 2, onde a pressão do refrigerante na entrada do evaporador aumenta e, consequentemente, sua temperatura também.

Conclui-se que como a condutância é mantida constante, quanto maior for a diferença de temperatura entre a água e o refrigerante, melhor a capacidade do evaporador. Uma vez que a 
temperatura do refrigerante aumentou e a temperatura da água está mantida constante $\left(30^{\circ} \mathrm{C}\right)$, diminui-se esta diferença de temperatura ao diminuir a vazão mássica.
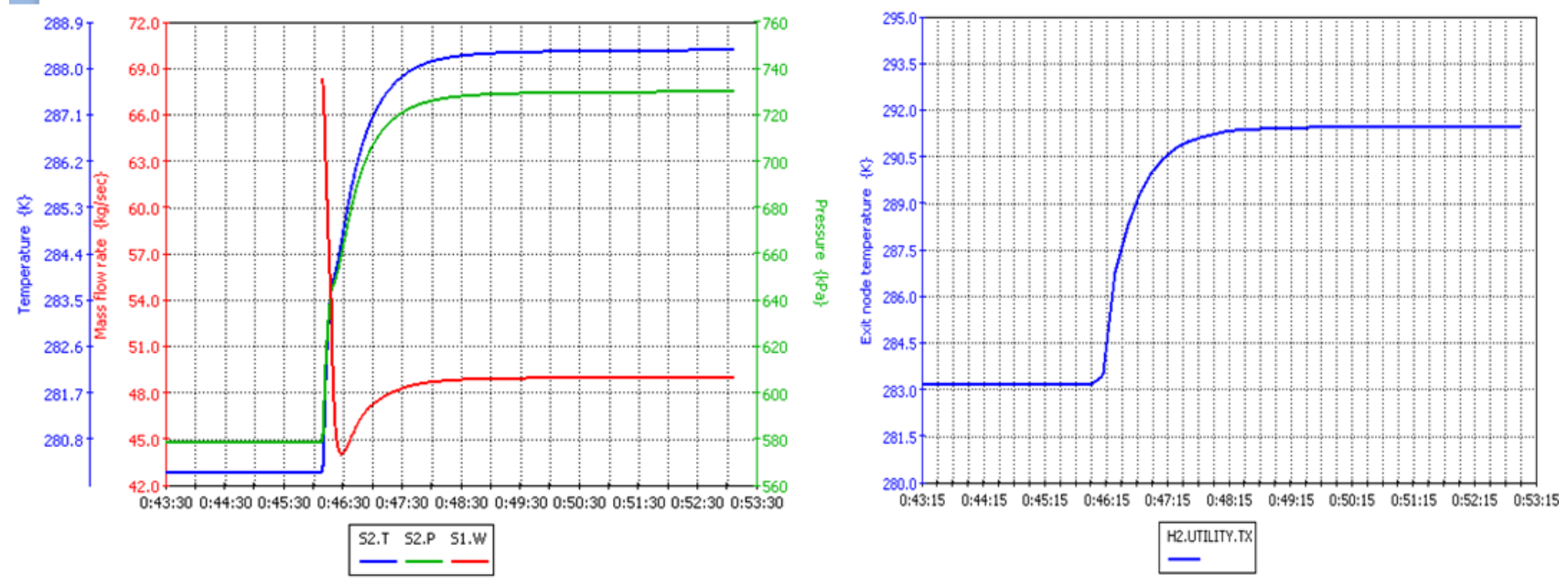

Figura 2 - Perfil de pressão e vazão mássica resultantes da diminuição da vazão mássica e comportamento da variável controlada.

Ao abrir a válvula XV1 simulando vazamento no circuito, observa-se a diminuição gradual do tráfego de vapor no circuito, bem como as pressões. À medida que o fluxo de refrigerante aumenta, o nível de líquido no tanque F1 diminui assim como o fluxo do vaporizador $\mathrm{H} 3$.

Observa-se que a diminuição da pressão e do fluxo ao longo de todo o ciclo permanece de forma constante, como mostra a Figura 3, até que haja alguma interrupção no vazamento. Se o vazamento não for interrompido, o compressor entrará em surto por falta de fluxo de sucção.
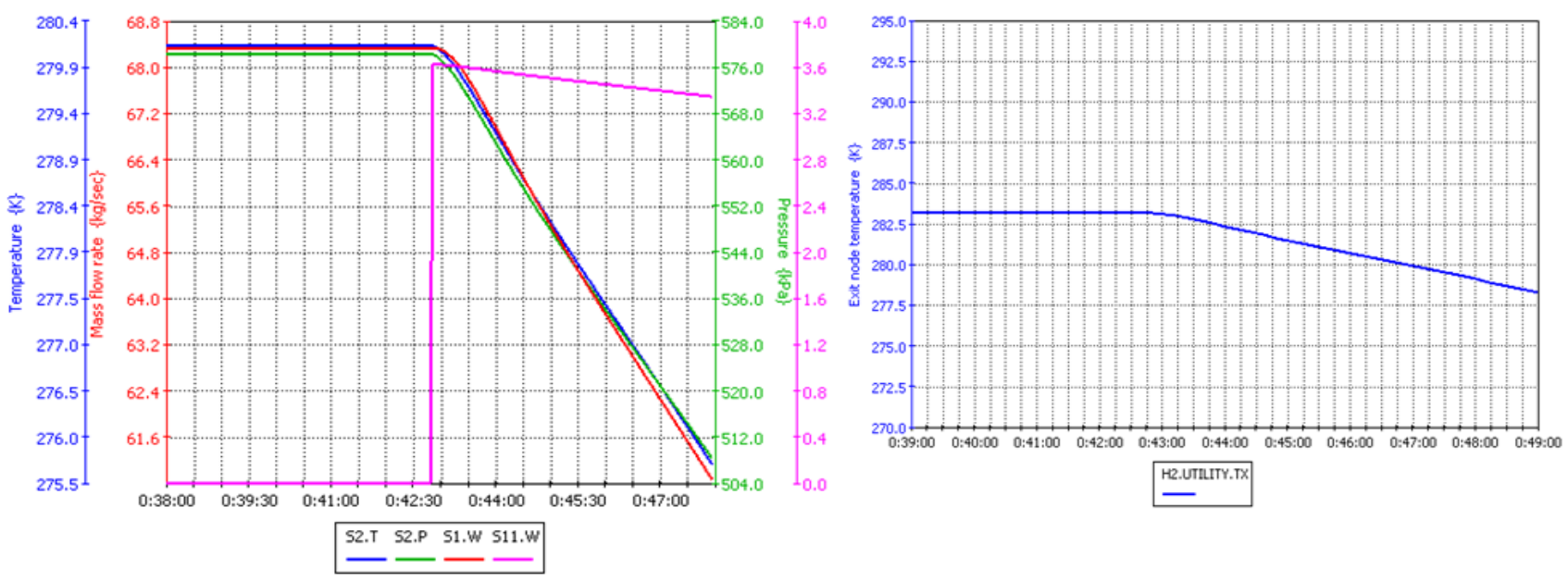

Figura 3 - Perfil de pressão e vazão resultantes do vazamento de refrigerante e comportamento da variável controlada. 


\subsection{Guideline do Controlador PID}

Para a análise das três ações do controlador PID, escolheu-se como variável manipulada a temperatura da água na entrada do evaporador, controlando desta forma a temperatura da água na saída do evaporador. $\mathrm{O}$ controle de desempenho do sistema foi medido pela aplicação de uma função degrau $\left(10^{\circ} \mathrm{C}\right.$ positivos $)$, em seguida foi medida a resposta da variável de processo.

A Figura 4 apresenta o comportamento da variável controlada em relação ao setpoint através da ação proporcional.

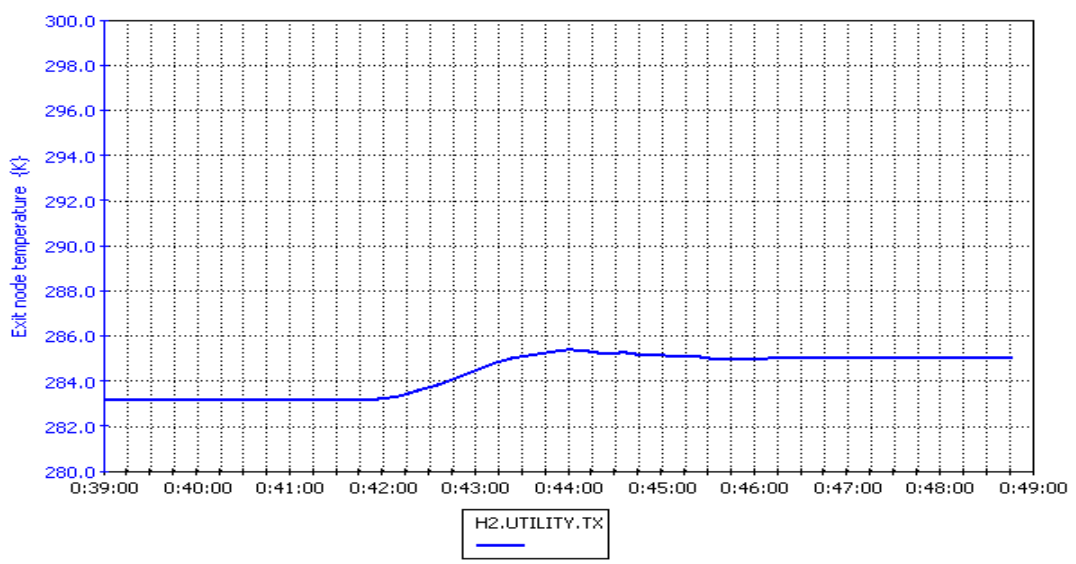

Figura 4 - Comportamento da variável controlada em relação ao setpoint.

Pode-se perceber a característica principal do controlador proporcional, que é a de não conseguir "zerar" o desvio do setpoint, deixando um erro residual (offset). Foi perceptível que com a banda proporcional grande, o processo estabiliza, porém muito abaixo do setpoint. Com a diminuição da banda proporcional, a estabilização ocorre mais próxima do setpoint, mas uma redução excessiva da banda proporcional pode levar o processo à instabilidade (oscilação). Esse ajuste da banda proporcional fez parte da sintonia do controle.

O processo se estabiliza acima do setpoint, em um tempo de aproximadamente 4,0 minutos. $\mathrm{O}$ maior pico atinge o valor de $285,6^{\circ} \mathrm{C}$, ou seja, $2,6^{\circ} \mathrm{C}$ acima do valor desejado.

A Figura 5 apresenta o comportamento da variável controlada em relação ao setpoint através da ação proporcional e integral. 


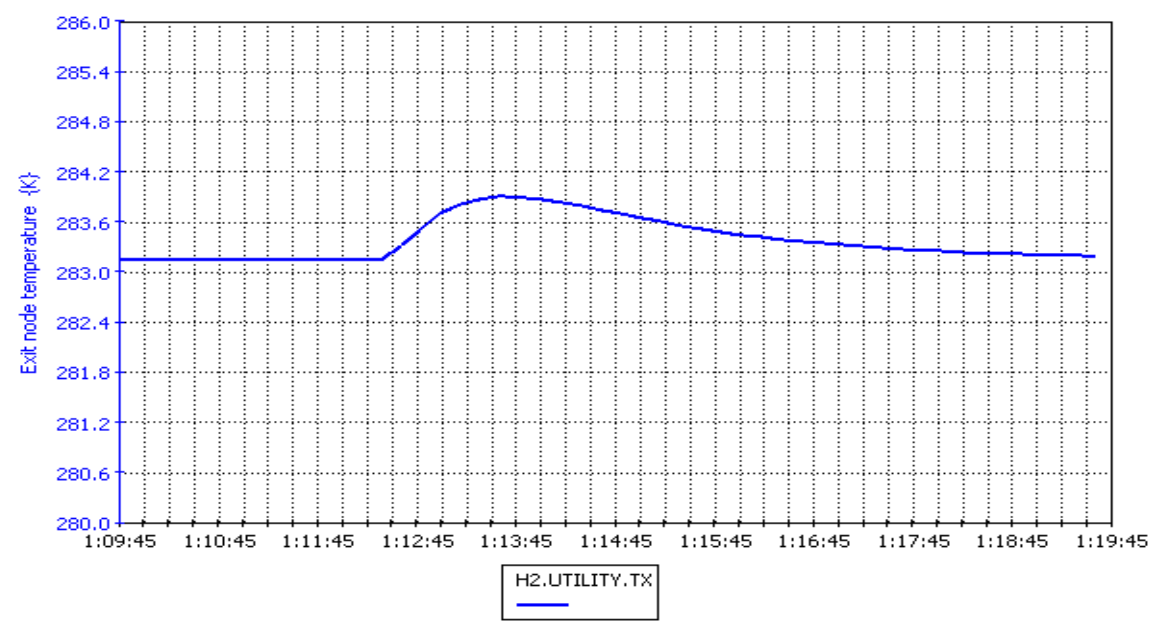

Figura 5 - Comportamento da variável controlada em relação ao setpoint.

A necessidade de adicionar ação integral foi devido à busca por precisão no controle da temperatura da água gelada. Nessas situações, offsets grandes podem gerar problemas operacionais significativos. Eliminando-os via ação integral, conseguem-se benefícios significativos.

Neste caso, o processo se estabiliza muito próximo do setpoint, em um tempo de aproximadamente 5 minutos. $\mathrm{O}$ maior pico atinge o valor de $284,0^{\circ} \mathrm{C}$, ou seja, apenas $1,0^{\circ} \mathrm{C}$ acima do valor desejado.

A Figura 6 apresenta o comportamento da variável controlada em relação ao setpoint através da ação proporcional, integral e derivativa.

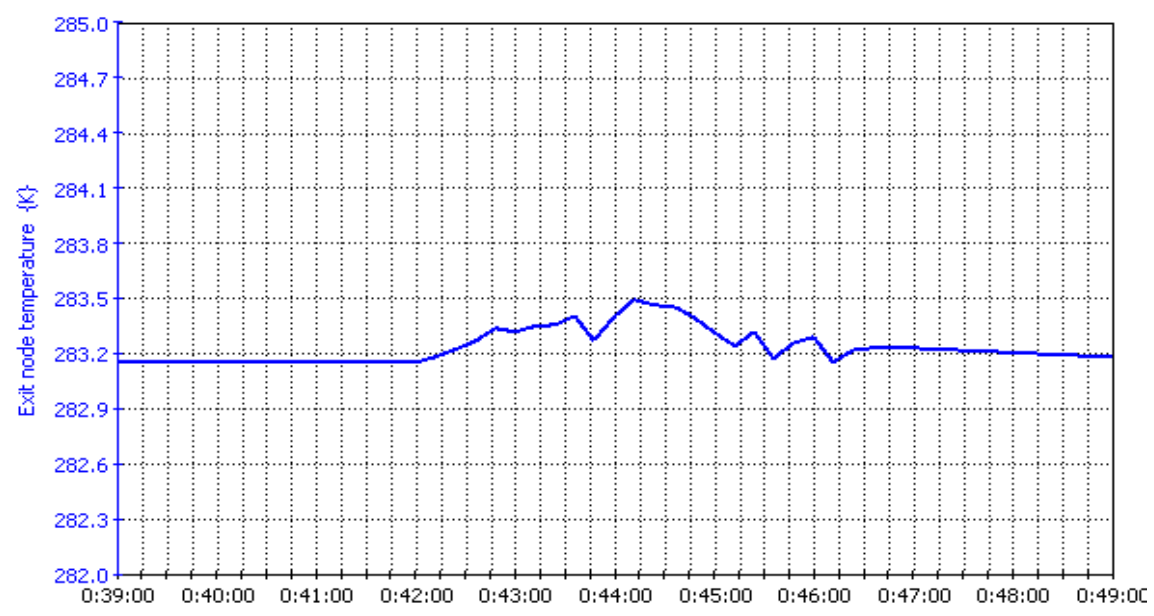

Figura 6 - Comportamento da variável controlada em relação ao setpoint. 
Através da Figura 6 é possível observar as características de um bom controle PID, desempenho estável, resposta rápida e pequeno desvio de estado estacionário. As características da onda de resposta mostram o tempo de subida menor que 20 segundos e o percentual overshoot de aproximadamente 0,14 . É perceptível que o maior pico atinge o valor de $283,5^{\circ} \mathrm{C}$, ou seja, apenas $0,5^{\circ} \mathrm{C}$ acima do valor desejado.

\section{CONCLUSÕES}

Os resultados obtidos mostram que com o uso do controlador PID a variável é corrigida da melhor maneira possível, quando comparado aos controladores P e PI.

Concluiu-se também a partir dos resultados, que o fluxo de fluido refrigerante do vaporizador e a vazão mássica do refrigerante são fatores que afetam a eficiência de refrigeração e, como observado, a carga correta de fluido refrigerante é um fator determinante para que o sistema opere sem danos.

É preciso ajustar cada parâmetro $\mathrm{K}_{\mathrm{p}}, \mathrm{T}_{\mathrm{i}}$ e $\mathrm{K}_{\mathrm{d}}$ dentro da área desempenho estável. Os fatores de ganhos ótimos do PID variam de condição para condição e, de sistema para sistema. Isso significa que é necessário definir esses parâmetros, levando em conta as características individuais de controle do seu sistema particular.

\section{REFERÊNCIAS}

ASTRÖM, K.; HÄGGLUND, T. The future of PID control. Control Engineering Practice, v. 9, p. 1163-1175, 2001.

BISSEL, C. C. Control Engineering. London: Chapman \& Hall, 1993.

CORRIPIO, A. B. Tuning of Industrial Control Systems, 1990.

DOSSAT, B. J. Princípios de Refrigeração, 2004.

LUYBEN, W.L. Process Modeling, Simulation and Control for Chemical Engineers, 1996.

QIN, S.; BADGWELLl, T. A Survey of Industrial Model Predictive Control Technology, Control Eng. Practice, v. 11, p. 733-764, 2003.

SKOGESTAD, S. Simple Analytic Rules for Model Reduction and PID Controller Tuning. Journal of Process Control, v. 13, p. 291-309, 2003. 